\title{
Annihilation of Methicillin-resistant Staphylococcus aureus via
}

\section{Photobleaching of Staphyloxanthin}

Pu-Ting Dong ${ }^{1}$, Haroon Mohammad ${ }^{2}$, Xiaoyu Wang ${ }^{3}$, Jie Hui ${ }^{4}$, Junjie Li ${ }^{5}$, Lijia Liang ${ }^{6}$, Mohamed N. Seleem ${ }^{2 *}$, and Ji-Xin Cheng ${ }^{1,5,7,8^{*}}$

1. Department of Chemistry, Boston University, Boston, MA 02215, USA

2. Department of Comparative Pathobiology, College of Veterinary Medicine, Purdue University, West Lafayette, IN 47907, USA

3. Molecular Biology, Cell Biology \& Biochemistry, Boston University, Boston, MA 02215, USA

4. Department of Physics and Astronomy, Purdue University, West Lafayette, IN 47907, USA

5. Department of Electrical and Computer Engineering, Boston University, Boston, MA 02215, USA

6. State Key Laboratory of Supramolecular Structure and Materials, Institute of Theoretical Chemistry, Jilin University, Changchun 130012, China

7. Department of Biomedical Engineering, Boston University, Boston, MA 02215, USA

8. Photonics Center, Boson University, Boston, MA 02215, USA

* To whom correspondence should be addressed: Ji-Xin Cheng (jxcheng@bu.edu) and Mohamed N. Seleem (mseleem@ purdue.edu). 
Given that the dearth of new antibiotic development loads an existential burden on successful infectious disease therapy ${ }^{1}$, health organizations are calling for alternative approaches to combat methicillin-resistant Staphylococcus aureus (MRSA) infections. Here, we report a drug-free photonic approach to eliminate MRSA through photobleaching of staphyloxanthin, an indispensable membrane-bound antioxidant of $S$. aureus ${ }^{2-5}$. The photobleaching process, uncovered through a transient absorption imaging study and quantitated by absorption spectroscopy and mass spectrometry, decomposes staphyloxanthin and sensitizes MRSA to reactive oxygen species attack. Consequently, staphyloxanthin bleaching by low-level blue light eradicates MRSA synergistically with external or internal reactive oxygen species. The effectiveness of this synergistic therapy is validated in MRSA culture, MRSA-infected macrophage cells, S. aureus biofilms, and a mouse wound infection model. Collectively, these findings highlight broad applications of staphyloxanthin photobleaching for treatment of MRSA infections.

Staphylococcus aureus causes a variety of diseases ranging from skin and soft tissue infections to life-threatening septicemia ${ }^{6-9}$. Moreover, $S$. aureus has acquired resistance to multiple antibiotic classes that were once effective ${ }^{10}$. A classic example is the emergence of clinical isolates of methicillin-resistant Staphylococcus aureus (MRSA) strains in the 1960s that exhibited resistance to $\beta$-lactam antibiotics ${ }^{11-13}$. More recently, strains of MRSA have exhibited reduced susceptibility to newer antibiotics such as daptomycin and antibiotics deemed agents of last resort such as vancomycin and linezolid ${ }^{14,15}$. Besides the acquired resistance through mutational inactivation, MRSA develops other strategies, e.g. residing inside host immune cells or forming biofilms, to evade the effect of antibiotics. Those strategies pose an appalling challenge to the successful therapy for MRSA infections. 
Initially we attempted to differentiate MRSA from methicillin-susceptible S. aureus by transient absorption imaging (see Methods and Supplementary Fig. 1) of their intrinsic chromophores. Intriguingly, once the cultured S. aureus was placed under the microscope, the strong signal measured at zero delay between the 520-nm pump and 780-nm probe pulses quickly attenuated over second time scale (Fig. 1a and Supplementary Video 1). We hypothesized that a specific chromophore in $S$. aureus is prone to photobleaching under the abovementioned setting. To verify the photobleaching phenomenon, we fitted the time-course curve with a photobleaching $\operatorname{model}^{16}$ (Fig. 1b):

$$
y=y_{0}+A * \frac{\exp \left(-\frac{t}{\tau_{1}}\right)}{1+\frac{\tau_{1}}{\tau_{2}} *\left(1-\exp \left(-\frac{t}{\tau_{1}}\right)\right)}
$$

, where $t$ is the duration of light irradiation, $\mathrm{y}$ is the signal intensity, $y_{0}$ and $\mathrm{A}$ are constants, $\tau_{1}$ and $\tau_{2}$ are the time constants for the first- and second-order bleaching, respectively. The firstorder bleaching occurs at low concentration of chromophores (singlet oxygen involved, $\tau_{2}=\infty$ ). The second-order bleaching takes place when quenching within high-concentration surrounding chromophores dominates $\left(\tau_{1}=\infty\right.$, Supplementary Fig. 2$)$. Derivation of equation (1) is detailed in Methods. Strikingly, this photobleaching model fitted well the raw time-course curve $\left(\tau_{1}=\infty\right.$, $\left.\tau_{2}=0.15 \pm 0.02 \mathrm{~s}, \mathrm{R}^{2}=0.99\right)$. Moreover, oxygen depletion $\left(\mathrm{Na}_{2} \mathrm{~S}_{2} \mathrm{O}_{4}\right.$ : oxygen scavenger $)$ showed negligible effect on the bleaching speed $\left(\tau_{2}=0.14 \pm 0.01 \mathrm{~s}\right.$, Supplementary Fig. 3a). The same phenomenon was observed in methicillin-susceptible S. aureus (Supplementary Fig. 3b). Collectively, these data support a second-order photobleaching process.

Next, we aimed to deduce the specific chromophore inside $S$. aureus that accounts for the observed photobleaching phenomenon. It is known that carotenoids are photosensitive due to the 
conjugated $\mathrm{C}=\mathrm{C}$ bonds ${ }^{17,18}$. Therefore, we hypothesized that staphyloxanthin (STX), a carotenoid pigment residing in the cell membrane of $S$. aureus, underwent photobleaching in our transient absorption study. To test this hypothesis, we treated MRSA with naftifine, a FDA-approved antifungal drug that blocks the synthesis of $\mathrm{STX}^{3}$. The treated MRSA exhibited lower signal intensity (Fig. 1c) and slower photobleaching speed (Fig. 1d). Specifically, $\tau_{2}$ of naftifine-treated $\operatorname{MRSA}(0.39 \pm 0.07 \mathrm{~s})$ is 2.5 times of that of MRSA $(0.15 \pm 0.02 \mathrm{~s})$, in consistence with secondorder photobleaching. Furthermore, no transient absorption signal was observed in a $S$. aureus stain containing a mutation in dehydrosqualene synthase (CrtM) (Fig. 1e) that is responsible for STX biosynthesis ${ }^{19}$. To avoid the systematic error aroused by single bacterium measurement, we repeated the same analysis using bacterial colonies. It turned out that CrtM-mutant colony (Fig. 1f,h) only exhibited background induced by cross-phase modulation ${ }^{20}$, whereas the MRSA colony showed a sharp contrast against the background (Fig. 1g) and a fast photobleaching decay (Fig. 1h). Taken together, these data confirm that STX in S. aureus accounts for the observed photobleaching.

In the transient absorption study, when changing 520-nm pump irradiance while fixing 780-nm probe intensity, both signal intensity and $\tau_{2}$ changed drastically (Supplementary Fig. 4a,c), whereas the alteration of probe irradiance only affected the transient absorption signal intensity but not $\tau_{2}$ (Supplementary Fig. 4b,d). These findings collectively imply that photobleaching efficacy is highly dependent on the excitation wavelength (Supplementary Fig. 4e), which is consistent with the fact that photobleaching is grounded on the absorption of chromophore $^{21}$.

To find the optimal wavelength for bleaching STX, we measured the absorption spectrum of crude STX extract from S. aureus. The extract shows strong absorption in the window from 
$400 \mathrm{~nm}$ to $500 \mathrm{~nm}$ (Fig. 2a). Based on this result, we built a portable device composed of a blue light-emitting diode (LED) with central emission wavelength around $460 \mathrm{~nm}$ for wide-field bleaching of STX (Fig. 2a and Supplementary Fig. 5). We exposed the crude STX extract to blue light $(90 \mathrm{~mW})$ for different time intervals. Remarkably, the distinctive golden color of STX disappeared within 30-min exposure, whereas the control group under ambient light remained unchanged (Fig. 2b). Its absorption peak between 400 and $500 \mathrm{~nm}$ decreased dramatically over blue light exposure time (Fig. 2c). The optical density (OD) at $470 \mathrm{~nm}$ (from Fig. 2c) versus the blue light dose can be well fitted with equation (1) (Fig. 2d). Additionally, naftifine-treated or CrtM-mutant MRSA extracts were insensitive to blue light exposure, indicated by their nearly unchanged absorption spectra (Supplementary Fig. 6a-c). These findings collectively suggest that STX is prone to bleaching under blue light irradiance.

To quantitate the photobleaching process, we studied STX degradation induced by blue light irradiation by mass spectrometry (MS). Supplementary Fig. 7 presents the MS spectrum of S. aureus extract with $\mathrm{m} / \mathrm{z}$ ranging from 200 to $1000 \mathrm{eV}$ at a collision energy of $10 \mathrm{eV}$. An abundant peak appears at $\mathrm{m} / \mathrm{z}=721.5$, while a weaker peak at $\mathrm{m} / \mathrm{z}=819.5\left(\left[\mathrm{M}+\mathrm{H}^{+}\right]\right)$is consistent with the molecular weight of $\operatorname{STX}\left(\mathrm{M}_{\mathrm{w}}=818.5 \mathrm{~g} / \mathrm{mol}\right)$. To find out the relationship between $\mathrm{m} / \mathrm{z}=721.5$ and $\mathrm{m} / \mathrm{z}=819.5$, we gradually increased the collision energy from 0 to 20 eV. In Fig. 2e, the abundance of $\mathrm{m} / \mathrm{z}=721.5$ increases relative to that of $\mathrm{m} / \mathrm{z}=819.5$ with increasing collision energy, which indicates $\mathrm{m} / \mathrm{z}=721.5$ is a product ion from $\mathrm{m} / \mathrm{z}=819.5$. These data also prove that STX is the major species in S. aureus extract. When the collision energy was higher than $30 \mathrm{eV}, \mathrm{m} / \mathrm{z}=241.5$, a product of the precursor ion $\mathrm{m} / \mathrm{z}=721.5$, became dominant and presented as a stable marker (Fig. 2e). Thus, to accurately quantify the amount of STX versus blue light dose, we targeted the peak area in high-performance liquid chromatography 
(HPLC) spectra specifically from ion $\mathrm{m} / \mathrm{z}=241.5$ (Fig. 2f). Figure $2 \mathrm{~g}$ depicts the blue light bleaching dynamics of STX. Blue light exposure for $5 \mathrm{~min}\left(\right.$ dose: $\left.27 \mathrm{~J} / \mathrm{cm}^{2}\right)$ decomposed $90 \%$ of STX extracted from $3.29 \times 10^{9}$ colony-forming-units (CFU/mL) S. aureus (Fig. $2 \mathrm{~g}$ ), and a dose of $54 \mathrm{~J} / \mathrm{cm}^{2}$ bleached all the STX pigments (data not shown). In contrast, naftifine-treated and CrtM-mutant $S$. aureus extracts had negligible response to blue light exposure (Supplementary Fig. 6d-f).

Next, we employed time-of-flight MS/MS (see Methods) to elucidate how blue light decomposed STX. Different from the $\mathrm{m} / \mathrm{z}=819.5$ peak where STX locates in the HPLC spectra, STX crests at $\mathrm{m} / \mathrm{z}=841.5$ in the ultra-performance liquid chromatography (UPLC) spectra (Fig. 2h), which is an adjunct between STX and $\mathrm{Na}^{+}$. Degradation of STX would bolster the aggregation of chemical segments. Accordingly, we screened a patch of the products after STX photobleaching (Supplementary Fig. 8). In particular, the intensity of the peak at $\mathrm{m} / \mathrm{z}=643.5$ representing an adjunct between a STX segment with $\mathrm{H}^{+}$, significantly increased as blue light exposure elongates (Fig. 2i). Figure $2 \mathrm{j}$ illustrates how this segment is formed from breakdown of one $\mathrm{C}=\mathrm{C}$ bond in STX during blue light bleaching. We note that interpretation of other products (Supplementary Fig. 8a-i) necessitates further in-depth analysis.

Given STX is critical to the integrity of $S$. aureus cell membrane ${ }^{19}$, we questioned whether blue light alone could eradiate MRSA through bleaching STX. Blue light at 405-420 nm has been used for MRSA suppression ${ }^{22}$. Yet the efficacy is limited and the molecular mechanism remain elusive. Here, we show that STX is the molecular target of blue light irradiation. We find that increasing blue light dose steadily decreased the level of MRSA CFU (Fig. 3a). Moreover, MRSA was more sensitive to blue light exposure than the CrtM mutant (Supplementary Fig. 9). Nevertheless, the positive impact on the reduction of CFU did not improve when the blue light 
dose exceeded $216 \mathrm{~J} / \mathrm{cm}^{2}$ with 60-min exposure time (Fig. 3a). To investigate the reason, we continuously monitored the growth of MRSA in fresh medium after 10-min blue light exposure. Remarkably, MRSA exposed to blue light was able to recover and multiply after being cultured in medium (Fig. 3b). Therefore, photobleaching STX alone is not sufficient to kill MRSA completely.

Considering that STX serves as an indispensable antioxidant for MRSA, we then explored whether photobleaching of STX could sensitize MRSA to reactive oxygen species (ROS) such as hydrogen peroxide $\left(\mathrm{H}_{2} \mathrm{O}_{2}\right)$. We examined the viability of MRSA exposed to $\mathrm{H}_{2} \mathrm{O}_{2}$ after blue light exposure. When MRSA was treated with blue light (dose: $108 \mathrm{~J} / \mathrm{cm}^{2}$ ) followed by an increasing concentration of $\mathrm{H}_{2} \mathrm{O}_{2}$, a significant reduction $(p<0.001)$ in CFU was obtained (Fig. 3c). Strikingly, 108-J/cm² blue light exposure combined with $13.2 \mathrm{mM}$ of $\mathrm{H}_{2} \mathrm{O}_{2}$ (culture time: $20 \mathrm{~min}$ ) eradicated $10^{7} \mathrm{MRSA}$ CFU completely (Fig. 3c). Therefore, we hypothesized that blue light plus $\mathrm{H}_{2} \mathrm{O}_{2}$ work synergistically towards MRSA-killing. To verify this synergistic effect, we performed the same measurements at various blue light doses while fixing the concentration of $\mathrm{H}_{2} \mathrm{O}_{2}$ (Fig. 3d). Then we calculated the fractional concentration index using an established model for synergy evaluation (see Methods). A fractional concentration index of 0.45 was obtained, indicating a strong synergy between blue light and $\mathrm{H}_{2} \mathrm{O}_{2}$ in eradication of MRSA. Noteworthy, this treatment did not affect other species of staphylococci, such as S. epidermidis (Fig. 3e), that lacks carotenoids.

Studies dating back to the 1970s have demonstrated that MRSA is able to invade and survive inside mammalian cells, particularly within macrophages ${ }^{23}$. Though macrophages secrete small effector molecules, including ROS, bacteria including MRSA are capable of neutralizing these effector molecules by producing antioxidants such as $\mathrm{STX}^{2}$. Meanwhile, antibiotics are 
generally ineffective at clearing intracellular MRSA in large part due to the efflux of drug by phagocytic membrane, which poses an alarming threat to the host cells ${ }^{23}$. As we have demonstrated that blue light plus $\mathrm{H}_{2} \mathrm{O}_{2}$ kills MRSA synergistically, we wondered whether blue light could synergize with the ROS inside macrophage cells to eliminate intracellular MRSA as illustrated in Fig. 3f. To evaluate this point, we infected macrophage cells by incubation with MRSA for one hour. The infected macrophages were then exposed to 2-min blue light (dose: 48 $\mathrm{J} / \mathrm{cm}^{2}$ ) twice over a 6-hour interval. The macrophages were subsequently lysed to enumerate CFU of MRSA (spread plates shown in Fig. 3g). Figure $3 \mathrm{~h}$ compiled the statistical analysis of different groups. Notably, a nearly one- $\log _{10}$ reduction in CFU was found in the blue lighttreated group in comparison to the untreated. On the contrary, vancomycin was unable to eradicate intracellular MRSA. Additionally, we found that whole blood could eradicate most of MRSA after STX bleaching by blue light (Supplementary Fig.10). These findings collectively suggest that blue light is capable of assisting neutrophils to eradicate intracellular MRSA.

Besides residing inside host immune cells, S. aureus is capable of forming biofilms. Due to difficulties for antibiotics to penetrate the matrix of biofilm termed extracellular polymeric substance $^{24}$, bacterial biofilms present a significant source of treatment failure and recurring infection in patients ${ }^{24}$. Compared to antibiotics, an unparalleled advantage of our photobleaching therapy lies in the fact that photons can readily penetrate through a cell membrane or a biofilm, or even a layer of tissue. To explore whether STX bleaching could eradicate $S$. aureus inside biofilm, we grew biofilms on the bottom of glass dish and then applied blue light or daptomycin (positive control) to the biofilms. Supplementary Fig. 11 shows that blue light alone (dose: 360 $\mathrm{J} / \mathrm{cm}^{2}$ ) reduced $S$. aureus CFU by $80 \%$. Blue light (dose: $360 \mathrm{~J} / \mathrm{cm}^{2}$ ) plus $\mathrm{H}_{2} \mathrm{O}_{2}(13.2 \mathrm{mM}, 20$ min culture time) reduced $S$. aureus $\mathrm{CFU}$ by $92 \%$. In contrast, daptomycin $(5 \times$ minimum 
inhibitory concentration (MIC), 24-hour culture time) only reduced $S$. aureus $\mathrm{CFU}$ by $70 \%$. These results suggest an effective way to eradicate sessile bacterial cells inside biofilms.

The promising results obtained from the intracellular infection and biofilm studies led us to evaluate the efficacy of STX photobleaching in a MRSA-infected animal model. Skin infections such as diabetic foot ulceration and surgical site infections ${ }^{25}$ are common causes of morbidity in healthcare settings. Notably, S. aureus accounts for $40 \%$ of these infections ${ }^{26}$. To optimize the parameters for the in vivo experiment, we initially proved that 2-min blue light exposure (dose: $24 \mathrm{~J} / \mathrm{cm}^{2}$ ) could cause significant reduction in survival percent of MRSA (Supplementary Fig. 12a). Then, two times antimicrobial efficiency was obtained when cultured with $\mathrm{H}_{2} \mathrm{O}_{2}$ (20-min culture time, $13.2 \mathrm{mM}$ ) subsequently. Furthermore, 5-min culture time with $\mathrm{H}_{2} \mathrm{O}_{2}$ after 2-min blue light exposure $\left(24 \mathrm{~J} / \mathrm{cm}^{2}\right)$ effectively eliminated MRSA by $60 \%$ (Supplementary Fig. 12b), which facilitates us to apply treatment in MRSA-infected animal model.

To induce skin lesions in mice, we severely irritated mice skin (5 groups; 5 mice per group) by an intradermal injection containing $10^{8} \mathrm{CFU}$ of MRSA USA300 (Fig. 4a), the leading source of $S$. aureus induced skin and soft tissue infections in North America ${ }^{27}$. Sixty hours post injection, an open wound formed at the site of infection (Fig. 4b (top)). Topical treatments were subsequently administered to each group, twice daily for three consecutive days. All the treated groups appeared healthier compared to the control group (Fig. 4b (middle)). Then, mice were humanely euthanized and wounds were aseptically removed in order to quantify the burden of MRSA in wounds (see Methods). We further examined the physiological condition of the wounds. The untreated, fusidic acid-treated (positive control), and blue light-treated groups all showed the formation of pus below the wound, most likely due to inflammatory response. In 
contrast, mice receiving only $\mathrm{H}_{2} \mathrm{O}_{2}$ or blue light plus $\mathrm{H}_{2} \mathrm{O}_{2}$ treatment exhibited clean wounds that were free of purulent material, swelling, and redness around the edge of the wound (Fig. 4b (bottom)). Notably, the blue light dosage applied to treat mouse wound infection was below the ANSI safety limit for skin exposure ${ }^{28}$.

To quantify the anti-inflammatory effect, we evaluated a panel of cytokines present in the supernatant of homogenized tissues extracted from the wounds of mice. By analysis of the skin homogenate collected from the MRSA mice wound model, we found the highest percent of negative fold change from around 200 kinds of cytokines in the blue light plus $\mathrm{H}_{2} \mathrm{O}_{2}$-treated group compared to the other groups (Supplementary Table. 1). Noteworthy, the blue light plus $\mathrm{H}_{2} \mathrm{O}_{2}$-treated group demonstrates the highest ratio of decreased expression of these proinflammatory cytokines (Fig. 4c). Specifically, there is a significant decrease observed in key pro-inflammatory cytokines (TNF- $\alpha$, IL-1 $\alpha$, IL-2, IL-17, MIP-1 $\alpha$, MIP-1 $\beta$, LIX) compared to the untreated mice. Furthermore, there was decreased expression of vascular endothelial growth factor receptor 3 (VEGF R3) in samples obtained from the blue light plus $\mathrm{H}_{2} \mathrm{O}_{2}$-treated group compared to the untreated group. This marker is overexpressed in chronic inflammatory wounds thus resulting in impaired wound reconstruction ${ }^{29}$. These results support a significantly decreased inflammation in the wounds of mice treated with blue light plus $\mathrm{H}_{2} \mathrm{O}_{2}$.

In order to quantify the burden of MRSA in wounds, homogenized wound tissue solution was inoculated onto mannitol salt agar plates (MRSA specific). Remarkably, the blue light plus $\mathrm{H}_{2} \mathrm{O}_{2}$-treated group showed 1.5- $\log _{10}$ reduction of CFU compared to the untreated group (Fig. 4d). Statistical analysis of CFU from the blue light plus $\mathrm{H}_{2} \mathrm{O}_{2}$-treated group depicted significant MRSA reduction compared to other groups (Fig. 4e). Noteworthy, the blue light plus $\mathrm{H}_{2} \mathrm{O}_{2-}$ treated group showed one- $\log _{10}$ more reduction than the fusidic acid-treated group (Fig. $\left.4 \mathrm{e}\right)$. It is 
worth noting that pigment is a hallmark feature of multiple pathogenic microbes ${ }^{30}$. Taken together, our findings show the exciting potential of treating drug-resistant bacteria by exploiting the unique photochemistry of their intrinsic pigments. 


\section{References}

1 Munguia, J. \& Nizet, V. Pharmacological targeting of the host-pathogen interaction: alternatives to classical antibiotics to combat drug-resistant superbugs. Trends Pharmacol Sci 38, 473-488 (2017).

2 Liu, G. Y. et al. Staphylococcus aureus golden pigment impairs neutrophil killing and promotes virulence through its antioxidant activity. J Exp Med 202, 209-215 (2005).

3 Chen, F. F. et al. Small-molecule targeting of a diapophytoene desaturase inhibits S. aureus virulence. Nat Chem Biol 12, 174-179 (2016).

4 Liu, C. I. et al. A cholesterol biosynthesis inhibitor blocks Staphylococcus aureus virulence. Science 319, 1391-1394 (2008).

5 Garcia-Fernandez, E. et al. Membrane microdomain disassembly inhibits MRSA antibiotic resistance. Cell (2017).

6 Diekema, D. J. et al. Survey of infections due to Staphylococcus species: Frequency of occurrence and antimicrobial susceptibility of isolates collected in the United States, Canada, Latin America, Europe, and the Western Pacific region for the SENTRY Antimicrobial Surveillance Program, 1997-1999. Clin Infect Dis 32, S114-S132 (2001).

7 Lewis, K. \& Strandwitz, P. Microbiology: Antibiotics right under our nose. Nature 535, 501-502 (2016).

8 McAdow, M. et al. Preventing Staphylococcus aureus sepsis through the inhibition of its agglutination in blood. Plos Pathog 7, e1002307 (2011).

9 Gaupp, R., Ledala, N. \& Somerville, G. A. Staphylococcal response to oxidative stress. Front Cell Infect Mi 2, 1-19 (2012).

10 Geisinger, E. \& Isberg, R. R. Interplay between antibiotic resistance and virulence during disease promoted by multidrug-resistant bacteria. J Infect Dis 215, S9-S17 (2017).

11 Guignard, B., Entenza, J. M. \& Moreillon, P. Beta-lactams against methicillin-resistant Staphylococcus aureus. Curr Opin Pharmacol 5, 479-489 (2005).

12 Hiramatsu, K. et al. Multi-drug-resistant Staphylococcus aureus and future chemotherapy. $J$ Infect Chemother 20, 593-601 (2014).

13 Tang, S. S., Apisarnthanarak, A. \& Hsu, L. Y. Mechanisms of beta-lactam antimicrobial resistance and epidemiology of major community- and healthcare-associated multidrug-resistant bacteria. Adv Drug Deliver Rev 78, 3-13 (2014).

14 Baek, J. Y. et al. Genetic alterations responsible for reduced susceptibility to vancomycin in community-associated MRSA strains of ST72. J Antimicrob Chemoth 72, 2454-2460 (2017).

15 Smith, J. R. et al. Daptomycin in combination with ceftolozane-tazobactam or cefazolin against daptomycin-susceptible and -nonsusceptible Staphylococcus aureus in an in vitro, hollow-fiber model. Antimicrob Agents Ch 60, 3970-3975 (2016).

16 Stratonnikov, A. A., Meerovich, G. A. \& Loschenov, V. B. Photobleaching of photosensitizers applied for photodynamic therapy. Prog Biom O 1, 81-91 (2000).

17 Cogdell, R. J. \& Frank, H. A. How carotenoids function in photosynthetic bacteria. Biochim Biophys Acta 895, 63-79 (1987).

18 Kumar, B. N. V., Kampe, B., Rosch, P. \& Popp, J. Characterization of carotenoids in soil bacteria and investigation of their photodegradation by UVA radiation via resonance Raman spectroscopy. Analyst 140, 4584-4593 (2015).

19 Mishra, N. N. et al. Carotenoid-related alteration of cell membrane fluidity impacts Staphylococcus aureus susceptibility to host defense peptides. Antimicrob Agents Ch 55, 526-531 (2011).

20 Min, W. et al. Imaging chromophores with undetectable fluorescence by stimulated emission microscopy. Nature 461, 1105-1109 (2009). 
21 Galassi, L. Wavelength dependence of the time course of fluorescence enhancement and photobleaching during irradiation of ethidium bromide-stained nuclei. Eur J Histochem 44, 419432 (2000).

22 Dai, T. et al. Blue light eliminates community-acquired methicillin-resistant Staphylococcus aureus in infected mouse skin abrasions. Photomedicine and laser surgery 31, 531-538 (2013).

23 Lehar, S. M. et al. Novel antibody-antibiotic conjugate eliminates intracellular S. aureus. Nature 527, 323-328 (2015).

24 Costerton, J. W., Stewart, P. S. \& Greenberg, E. P. Bacterial biofilms: A common cause of persistent infections. Science 284, 1318-1322 (1999).

25 Ager, S. \& Gould, K. Clinical update on linezolid in the treatment of Gram-positive bacterial infections. Infect Drug Resist 5, 87-102 (2012).

26 Weigelt, J. et al. Linezolid versus vancomycin in treatment of complicated skin and soft tissue infections. Antimicrob Agents Chemother 49, 2260-2266 (2005).

27 Immergluck, L. C. et al. Risk of skin and soft tissue infections among children found to be Staphylococcus aureus MRSA USA300 carriers. Western Journal of Emergency Medicine 18, 201-212 (2017).

28 American National Standard for Safe Use of Lasers, ANSI Z136.1. Laser Institute of America. (2014).

29 Paavonen, K., Puolakkainen, P., Jussila, L., Jahkola, T. \& Alitalo, K. Vascular endothelial growth factor receptor-3 in lymphangiogenesis in wound healing. Am J Pathol 156, 1499-1504 (2000).

30 Liu, G. Y. \& Nizet, V. Color me bad: microbial pigments as virulence factors. Trends Microbiol 17, 406-413 (2009). 


\section{Acknowledgements}

We thank Dr. George Y. Liu of Cedars-Sinai Medical Center for providing CrtM mutant strain and Dr. Bruce Copper of Purdue University for help in the mass spectrometry study. Funding: This work was supported by a Keck Foundation Science \& Engineering Grant to J.-X.C.

\section{Author contributions}

P.-T.D. and X.W. made the accidental discovery. J.-X.C. conceived the concept of photobleaching therapy. J.H. and P.-T.D. mathematically analyzed the photobleaching. P.-T.D. and X.W. did the in vitro experiments. P.-T.D. and L.L. performed the biofilm experiment. H.M. and P.-T.D. carried out the intracellular study and the mice wound infection study. J.L. guided the synergy analysis. P.-T.D. and J.H. analyzed the data. M.N.S. designed the animal study and provided cytokine analysis. J.-X.C. and P.-T.D. co-wrote the paper.

\section{Competing financial interest}

J.-X.C has a financial interest in Vibronix Inc. 


\section{Materials and methods}

\section{$\underline{\text { Transient absorption microscope }}$}

An optical parametric oscillator synchronously pumped by a femtosecond pulsed laser generates pump $(1040 \mathrm{~nm})$ and probe $(780 \mathrm{~nm})$ pulse trains (Supplementary Fig. 1). The pump $(1040 \mathrm{~nm})$ is then frequency-doubled (second harmonic generation (SHG) process) to $520 \mathrm{~nm}$ through a barium borate (BBO) crystal. Temporal delay between the pump and probe pulses is controlled through a motorized delay stage. The pump beam intensity is modulated with an acousto-optic modulator (AOM). The intensity of each beams is adjustable through the combination of a half-wave plate (HWP) and a polarization beam splitter (PBS). Thereafter, pump and probe beams are collinearly combined and directed into a lab-built laser-scanning microscope. Through the nonlinear process in the sample, the modulation of pump beam is transferred to the un-modulated probe beam. Computer-controlled scanning galvo mirrors are used to scan the combined laser beams in a raster scanning approach to create microscopic images. The transmitted light is collected by an oil condenser. Subsequently, the pump beam is spectrally filtered by an optical filter and the transmitted probe intensity is detected by a photodiode. A phase-sensitive lock-in amplifier then demodulates the detected signal. Therefore, pump-induced transmission changes in probe beam versus the temporal delay can be measured. This change over time delay shows different time-domain signatures of a chromophore, thus offering the origin of the chemical contrast.

$\underline{\text { Portable blue light photobleaching apparatus }}$ 
The apparatus is comprised of three parts: a blue light LED (M470L3, Thorlabs), an adjustable collimator (SM1P25-A, Thorlabs), and a power controller (LEDD1B, Thorlabs). The blue light LED has a central emission wavelength of $460 \mathrm{~nm}$ with a full width at half maximum of $30 \mathrm{~nm}$. The beam size is adjustable through the collimator (SM1P25-A, Thorlabs). The maximal power of the blue light LED is $200 \mathrm{~mW} / \mathrm{cm}^{2}$.

\section{Carotenoids extraction from S. aureus and acquisition of absorption spectrum}

The pigment extraction protocol was adapted from a previous report ${ }^{2}$. Briefly, $100 \mu \mathrm{L}$ of bacteria solution supplemented with $1900 \mu \mathrm{L}$ sterile Luria-Bertani (LB) broth was cultured for 24 hours with shaking (speed of $250 \mathrm{rpm}$ ) at $37{ }^{\circ} \mathrm{C}$. The suspension was subsequently centrifuged for two minutes at 7,000 rpm, washed once, and re-centrifuged. The pigment was extracted with $200 \mu \mathrm{L}$ methanol at $55^{\circ} \mathrm{C}$ for 20 minutes. Pigments from the $\mathrm{CrtM}$ mutant were extracted by the same method. The protocol for the treatment of $S$. aureus with naftifine was adapted from a published report ${ }^{3}$. Bacteria were cultured with $0.2 \mathrm{mM}$ naftifine for 24 hours at $37^{\circ} \mathrm{C}$ with the shaking speed of $250 \mathrm{rpm}$. The extraction procedure was the same as described above. The extracted solutions were subsequently exposed to blue light $(90 \mathrm{~mW}$, aperture: $1 \mathrm{~cm}$ $\times 1 \mathrm{~cm})$ at different time intervals $(0 \mathrm{~min}, 5 \mathrm{~min}, 10 \mathrm{~min}, 20 \mathrm{~min})$. Absorption spectra of the above solutions were obtained by a spectrometer (SpectraMax, M5).

\section{Mass spectrometry}

To study the photobleaching effect on STX, we extracted crude STX from S. aureus and exposed the extract to blue light using the procedure described above. The separation was 
performed on an Agilent Rapid Res 1200 HPLC system. The HPLC-MS/MS system consisted of a quaternary pump with a vacuum degasser, thermostated column compartment, auto-sampler, data acquisition card, and triple quadrupole (QQQ) mass spectrometer (Agilent Technologies, Palo Alto, CA, USA). An Agilent (ZORBAX) SB-C8 column (particle size: $3.5 \mu \mathrm{m}$, length: 50 $\mathrm{mm}$, and internal diameter: $4.6 \mathrm{~mm}$ ) was used at a flow rate of $0.8 \mathrm{~mL} / \mathrm{min}$. The mobile phase A was water with $0.1 \%$ formic acid and mobile phase B was acetonitrile with $0.1 \%$ formic acid. The gradient increased linearly as follows: $5 \% \mathrm{~B}$, from one to five $\mathrm{min}$; $95 \% \mathrm{~B}$ from five to six min, and 5\% B. Column re-equilibration was 6-10 min, 5\% B. The relative concentration of STX was quantified using MS/MS utilizing the Agilent 6460 QQQ mass spectrometer with positive electrospray ionization. Quantitation was based on multiple reaction monitoring. Mass spectra were acquired simultaneously using electrospray ionization in the positive modes over the $\mathrm{m} / \mathrm{z}$ range of 100 to 1000 . Nitrogen was used as the drying flow gas.

In order to understand how STX degrades when exposed to blue light, an Agilent 6545 quadrupole time-of-flight (Q-TOF) (Agilent, Santa Clara, CA, USA) was exploited to conduct the separation and quantification steps. This ultra-performance liquid chromatography (UPLC)MS/MS utilized an Agilent (ZORBAX) SB-C8 column (particle size: $3.5 \mu \mathrm{m}$, length: $50 \mathrm{~mm}$, and internal diameter: $4.6 \mathrm{~mm}$ ) to conduct the separation at a flow rate of $0.8 \mathrm{~mL} / \mathrm{min}$. The relative concentration of STX was quantified using MS/MS utilizing the Agilent 6545 Q-TOF MS/MS with positive electrospray ionization. The mobile phase was composed of water (A) and acetonitrile (B). The gradient solution with a flow rate of $0.8 \mathrm{~mL} / \mathrm{min}$ was performed as follows: $85 \% \mathrm{~B}$, from 0 to $30 \mathrm{~min} ; 95 \% \mathrm{~B}$, from 30 to $31 \mathrm{~min} ; 85 \% \mathrm{~B}$, from 31 to $35 \mathrm{~min} ; 85 \% \mathrm{~B}$, after 35 min. The sample injection volume was $20 \mu \mathrm{L}$. The UPLC-MS/MS analysis was performed in positive ion modes in the $\mathrm{m} / \mathrm{z}$ range of $100-1100$. 
$\underline{\text { In vitro assessment of synergy between blue light and } \underline{\mathrm{H}}_{2} \underline{\mathrm{O}}_{2}}$

MRSA USA300 was cultured in sterile LB broth in a $37^{\circ} \mathrm{C}$ incubator with shaking (at 250 $\mathrm{rpm})$ until the suspension reached the logarithmic growth phase $\left(\mathrm{OD}_{600}=0.6\right)$. Thereafter, an aliquot $(20 \mu \mathrm{L})$ of the bacterial suspension was transferred onto a glass slide. Samples were exposed to blue light at different time lengths and variable light intensities. For groups treated with $\mathrm{H}_{2} \mathrm{O}_{2}$, bacteria were collected in either LB or phosphate buffered saline (PBS) supplemented with $\mathrm{H}_{2} \mathrm{O}_{2}$ at different concentrations $(0 \mathrm{mM}, 0.8 \mathrm{mM}, 1.6 \mathrm{mM}, 3.3 \mathrm{mM}, 6.6 \mathrm{mM}$, and $13.2 \mathrm{mM}$ ). The solutions were cultured for $20 \mathrm{~min}$. The solution was serially diluted in sterile PBS and transferred to LB plates in order to enumerate the viable number of MRSA CFU. Plates were incubated at $37^{\circ} \mathrm{C}$ for 24 hours before counting viable $\mathrm{CFU} / \mathrm{mL}$. Data are presented as viable MRSA CFU/mL and percent survival of MRSA CFU/mL in the treated groups.

Fluorescence mapping of live and dead S. aureus in biofilm

An overnight culture of $S$. aureus (ATCC 6538) was grown in a $37{ }^{\circ} \mathrm{C}$ incubator with shaking (at $250 \mathrm{rpm}$ ). Poly-D-lysine (Sigma Aldrich) was applied to coat the surface of glass bottom dishes (35 mm, In Vitro Scientific) overnight. The overnight culture of $S$. aureus was diluted (1:100) in LB containing 5\% glucose and transferred to the glass bottom dishes. The plates were incubated at $37^{\circ} \mathrm{C}$ for $24-48$ hours in order to form mature biofilm. Thereafter, the media was removed and the surface of the dish was washed gently with sterile water to remove planktonic bacteria. Plates were subsequently treated with blue light alone $\left(200 \mathrm{~mW} / \mathrm{cm}^{2}, 30\right.$ min), $\mathrm{H}_{2} \mathrm{O}_{2}$ (13.2 mM, 20 minutes) alone, or a combination of blue light and $\mathrm{H}_{2} \mathrm{O}_{2}$. Groups 
receiving $\mathrm{H}_{2} \mathrm{O}_{2}$ were quenched through addition of $0.5 \mathrm{mg} / \mathrm{mL}$ catalase (Sigma Aldrich, $50 \mathrm{mM}$, $\mathrm{pH}=7$ in potassium buffered solution). After treatment, biofilms were immediately stained with fluorescence dyes, as follows.

To confirm the existence of biofilm on the glass bottom surface, a biofilm matrix stain $\left(\right.$ SYPRO $^{\circledR}$ Ruby Biofilm Matrix Stain, Invitrogen) was utilized. Biofilms were stained with the live/dead biofilm viability kit (Invitrogen) for 30 minutes to quantify the survival percent of $S$. aureus in the biofilm after treatment. The biofilms were washed with sterile water twice and then imaged using a fluorescence microscope (OLYMPUS BX51, objective: 60×, oil immersion, NA = 1.5). Two different excitation channels (live: FITC; dead: Texas Red) were utilized in order to map the ratio of live versus dead cells within the biofilm. The acquired images were analyzed by ImageJ. Statistical analysis was conducted via a two-paired $t$-test through GraphPad Prism 6.0 (GraphPad Software, La Jolla, CA).

Intracellular MRSA infection model

Murine macrophage cells (J774) were cultured in Dulbecco's Modified Eagle Medium (DMEM) supplemented with $10 \%$ fetal bovine serum (FBS) at $37{ }^{\circ} \mathrm{C}$ with $\mathrm{CO}_{2}(5 \%)$. Cells were exposed to MRSA USA400 at a multiplicity of infection of approximately 100:1. 1-hour postinfection, J774 cells were washed with gentamicin $(50 \mu \mathrm{g} / \mathrm{mL}$, for one hour) to kill extracellular MRSA. Vancomycin, at a concentration equal to $2 \mu \mathrm{g} / \mathrm{mL}(4 \times \mathrm{MIC}$, MIC: minimal inhibition concentration), was added to six wells. Six wells received blue light treatment twice (six hours between treatments) for two minutes prior to addition of DMEM $+10 \%$ FBS. Three wells were left untreated (medium + FBS) and three wells received dimethyl sulfoxide at a volume equal to 
vancomycin-treated wells. Twelve hours after the second blue light treatment, the test agents were removed; J774 cells were washed with gentamicin $(50 \mu \mathrm{g} / \mathrm{mL})$ and subsequently lysed using $0.1 \%$ Triton-X 100 . The solution was serially diluted in PBS and transferred to tryptic soy agar plates in order to enumerate the MRSA CFU present inside infected J774 cells. Plates were incubated at $37^{\circ} \mathrm{C}$ for 22 hours before counting viable $\mathrm{CFU} / \mathrm{mL}$. Data are presented as $\log _{10}(\mathrm{MRSA} \mathrm{CFU} / \mathrm{mL})$ in infected $\mathrm{J} 774$ cells in relation to the untreated control. The data was analyzed via a two-paired $t$-test, utilizing GraphPad Prism 6.0 (GraphPad Software, La Jolla, CA).

$\underline{\text { In vivo MRSA mice wound model }}$

All animal experiments were conducted following protocols approved by Purdue Animal Care and Use Committee (PACUC). To initiate the formation of a skin wound, five groups $(\mathrm{N}=$ 5) of eight-week-old female Balb/c mice (obtained from Harlan Laboratories, Indianapolis, IN, USA) were disinfected with ethanol (70\%) and shaved on the middle of the back (approximately a one-inch by one-inch square region around the injection site) one day prior to infection as described from a reported procedure (Ref. 31). To prepare the bacterial inoculum, an aliquot of overnight culture of MRSA USA300 was transferred to fresh tryptic soy broth and shaken at 37 ${ }^{\circ} \mathrm{C}$ until an $\mathrm{OD}_{600}$ value of $\sim 1.0$ was achieved. The cells were centrifuged, washed once with PBS, re-centrifuged, and then re-suspended in PBS. Mice subsequently received an intradermal injection $(40 \mu \mathrm{L})$ containing $2.40 \times 10^{9} \mathrm{CFU} / \mathrm{mL}$ MRSA USA300. An open wound formed at the site of injection for each mouse, $\sim 60$ hours post-infection.

Topical treatment was initiated subsequently with each group of mice receiving the following: fusidic acid (2\%, using petroleum jelly as the vehicle), $13.2 \mathrm{mM} \mathrm{H} \mathrm{O}_{2}(0.045 \%$, two-minute 
exposure), blue light (two-minute exposure, $24 \mathrm{~J} / \mathrm{cm}^{2}$ ), or a combination of blue light (twominute exposure) and $13.2 \mathrm{mM} \mathrm{H}_{2} \mathrm{O}_{2}$ (two-minute exposure). One group of mice was left untreated (negative control). Each group of mice receiving a particular treatment regimen was housed separately in a ventilated cage with appropriate bedding, food, and water. Mice were checked twice daily during infection and treatment to ensure no adverse reactions were observed. Mice were treated twice daily (once every 12 hours) for three days, before they were humanely euthanized via $\mathrm{CO}_{2}$ asphyxiation 12 hours after the last dose was administered. The region around the skin wound was lightly swabbed with ethanol (70\%) and excised. The tissue was subsequently homogenized in PBS. The homogenized tissue was then serially diluted in PBS before plating onto mannitol salt agar plates. Plates were incubated for at least 19 hours at $37{ }^{\circ} \mathrm{C}$ before viable MRSA CFU/mL were counted for each group. Outlier was removed based upon the Dixon Q Test. Data were analyzed via a two-paired $t$-test, utilizing GraphPad Prism 6.0 (GraphPad Software, La Jolla, CA).

$\underline{\text { Statistical analysis }}$

Data were present as mean values and its standard error of the mean. Statistical analysis was conducted through two-paired $t$-test. ${ }^{* * *}$ means significantly different with the $p$-value $<0.001$. ${ }^{* *}$ means significantly different with the $p$-value $<0.01$. ${ }^{*}$ means significantly different with the $p$-value $<0.05$. ns means no significance.

$\underline{\text { Human whole blood }}$ 
After photobleaching of MRSA by blue light, MRSA were either cultured in sterile PBS (control) or human whole blood (Innovative Research Inc., Novi, MI) for 9 hours. The efficacy was evaluated through enumerating CFU.

\section{$\underline{\text { Photobleaching model }}$}

To analyze the time-lapse transient absorption signals, we utilized a mathematical model which was originally used to depict the photobleaching of photosensitizers happening during a photodynamic process ${ }^{16}$ :

$$
\frac{d[C]}{d t}=-k_{1}[C][R]
$$

, where $t$ is the duration time, [C] is the concentration of chromophore (e.g., carotenoids in $S$.

aureus $), k_{1}\left(k_{1}=1 / \tau_{1}\right)$ is the rate constant of first-order photobleaching with $\tau_{1}$ being the firstorder photobleaching time constant, $[\mathrm{R}]$ is the concentration of active agents (the chromophores which have interaction with light):

$$
[R] \sim[R]_{0}+k_{2}[C]
$$

, where $k_{2}\left(k_{2}=1 /\left([C]_{0} * \tau_{2}\right)\right.$ is the rate constant of second-order photobleaching with $\tau_{2}$ being the second-order photobleaching time constant, $[R]_{0}$ is the initial concentration of the active agent, respectively. The combination of equation (1) and equation (2) leads to:

$$
\frac{d[C]}{d t}=-\frac{1}{\tau_{1}} *[C]-\frac{1}{\tau_{2} *[C]_{0}} *[C]^{2}
$$

The solution for equation (3) is:

$$
\frac{[C]_{t}}{[C]_{0}}=A * \frac{\exp \left(-\frac{t}{\tau_{1}}\right)}{1+\frac{\tau_{1}}{\tau_{2}} *\left(1-\exp \left(-\frac{t}{\tau_{1}}\right)\right.}
$$


, where A is a constant. When first-order photobleaching process pivots (usually happening for low concentration of chromophore and having the involvement of oxygen), $\tau_{2} \rightarrow \infty$, then equation (4) becomes:

$$
\frac{[C]_{t}}{[C]_{0}}=A * \exp \left(-\frac{t}{\tau_{1}}\right)
$$

, which is similar to first-order kinetic reaction. At this occasion, the photobleaching rate is linearly proportional to the concentration of chromophore. When second-order photobleaching process dominates (usually happening for high concentration of chromophore potentially through triplet-triplet annihilation), $\tau_{1} \rightarrow \infty$, then equation (4) becomes:

$$
\frac{[C]_{t}}{[C]_{0}}=A * \frac{1}{1+\frac{t}{\tau_{2}}}
$$

Under this condition, the photobleaching rate is proportional to the square of chromophore concentration. According to the fitting result, S. aureus belongs to second-order bleaching with $\tau_{1} \rightarrow \infty$

$\underline{\text { Synergistic antimicrobial effect analysis }}$

The synergy analysis is based on the calculation of fractional bactericidal concentration index (FBCI) (Ref. 32). Here, FBC stands for fractional bactericidal concentration and MBC is minimal bactericidal concentration. FBCI was calculated as follows: $\mathrm{FBC}$ of drug $\mathrm{A}=\mathrm{MBC}$ of drug $\mathrm{A}$ in combination with drug $\mathrm{B} / \mathrm{MBC}$ of drug $\mathrm{A}$ alone, $\mathrm{FBC}$ of drug $\mathrm{B}=\mathrm{MBC}$ of drug $\mathrm{B}$ in combination with drug $\mathrm{A} / \mathrm{MBC}$ of drug $\mathrm{B}$ alone, and $\mathrm{FBCI}$ index $=\mathrm{FBC}$ of drug $\mathrm{A}+\mathrm{FBC}$ of drug B. An FBCI of $\leq 0.5$ is considered to demonstrate synergy. Additive was defined as an FBCI of 1. 
Antagonism was defined as an FBCI > 4. Since $200 \mathrm{~mW} / \mathrm{cm}^{2}$ blue light did not kill 100\% MRSA after 1-hour exposure time, we have

FBC of drug A (blue light) $<\frac{0.12 \times 30 \times 60}{0.20 \times 60 \times 60}=0.30$

Since we found that $88 \mathrm{mM} \mathrm{H}_{2} \mathrm{O}_{2}$ is needed to eradicate all the bacteria, we have

FBC of drug $B\left(\mathrm{H}_{2} \mathrm{O}_{2}\right)=\frac{13.2 \mathrm{mM}}{88 \mathrm{mM}}=0.15$

Therefore, $\mathrm{FBCI}=\mathrm{FBC}$ of blue light $+\mathrm{FBC}$ of $\mathrm{H}_{2} \mathrm{O}_{2}<(0.30+0.15=0.45)$.

References (for Materials and Methods)

31. H. Mohammad, M. Cushman, M. N. Seleem, Antibacterial evaluation of synthetic thiazole compounds in vitro and in vivo in a methicillin-resistant Staphylococcus aureus (MRSA) skin infection mouse model. PLoS ONE 10, e0142321 (2015).

32. A. Horrevorts, M. Michel, K. Kerrebijn, Antibiotic interaction: interpretation of fractional inhibitory and fractional bactericidal concentration indices. European Journal of Clinical Microbiology \& Infectious Diseases 6, 502 (1987). 


\section{Figures and captions}
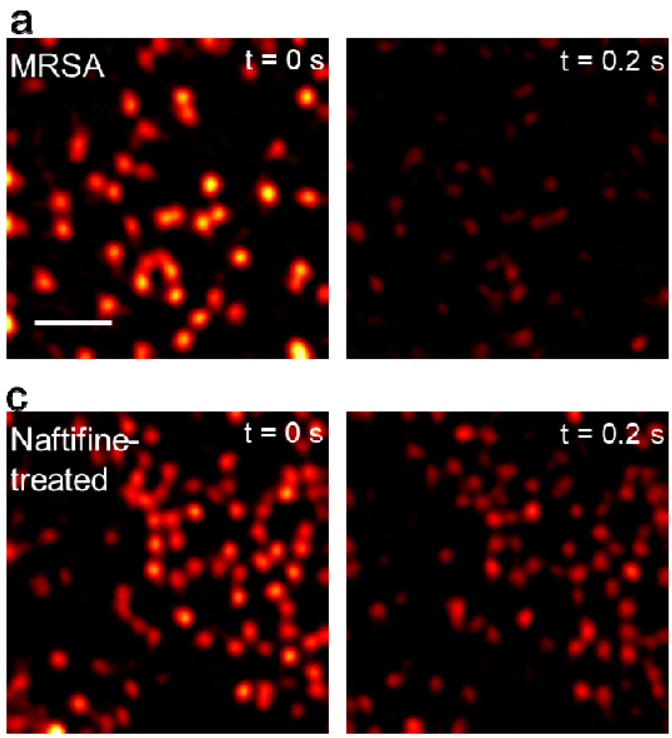

e

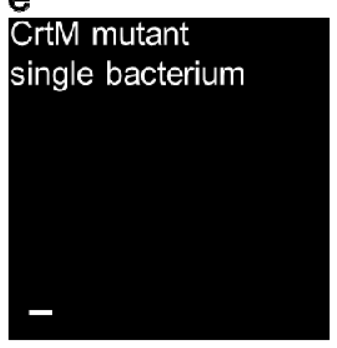

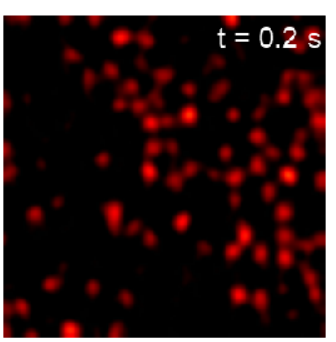

f

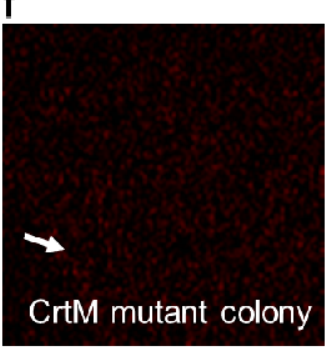

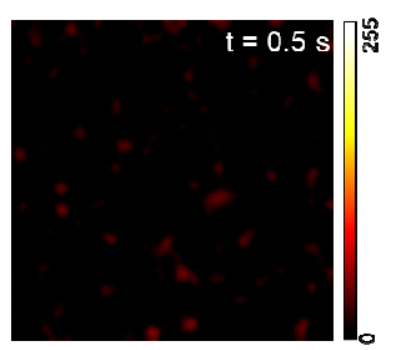
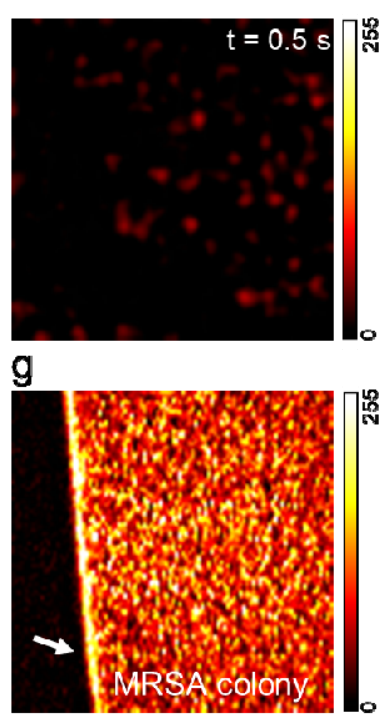
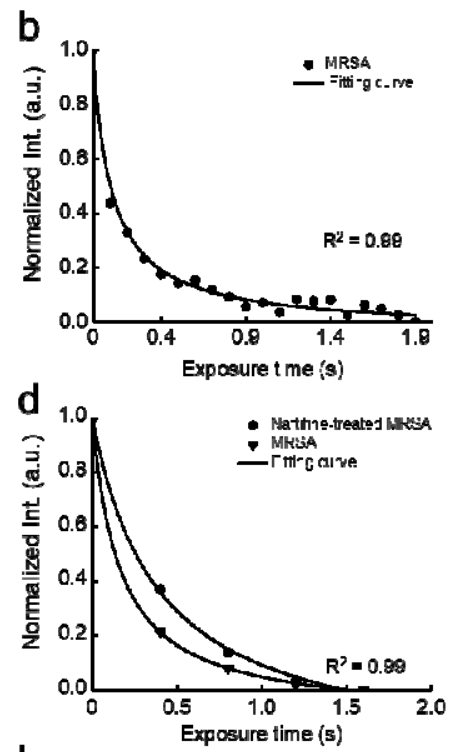

h

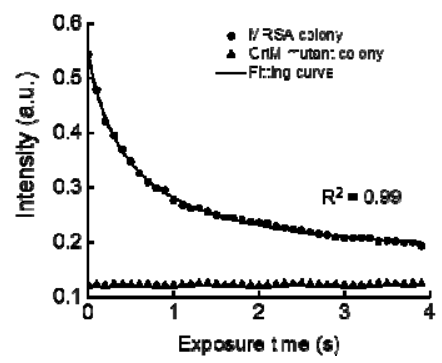

Fig. 1. Photobleaching of staphyloxanthin in MRSA uncovered by transient absorption microscopy. (a) Time-lapse images of MRSA. Scale bar, 5 m, applies to (a,c). (b) Representative normalized time-lapse signal from MRSA. (c) Time-lapse images of naftifinetreated MRSA. (d) Representative normalized time-lapse signals from MRSA and naftifinetreated MRSA. (e-g) Images of CrtM mutant, CrtM mutant colony, MRSA colony at $\mathrm{t}=0 \mathrm{~s}$, respectively. Scale bar, $20 \mathrm{~m}$, applies to (e-g). (h) Representative raw time-lapse signals from MRSA colony and CrtM mutant colony. White arrows indicate the interface between air and sample. Time-lapse signals were fitted by equation (1). The images are processed from the raw data with dynamic range of 0-255 through ImageJ. 


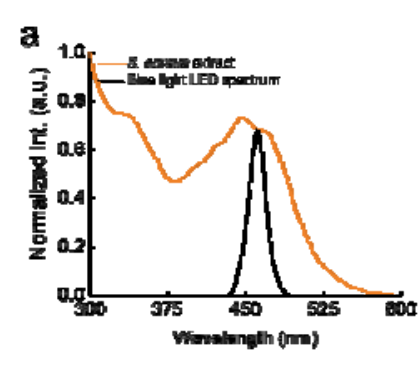

-

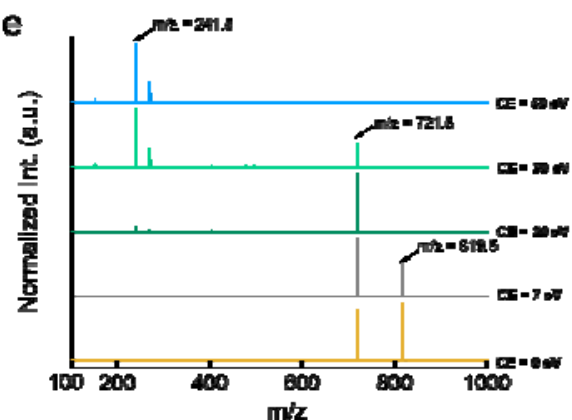

h

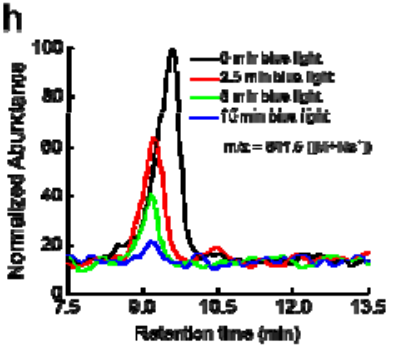

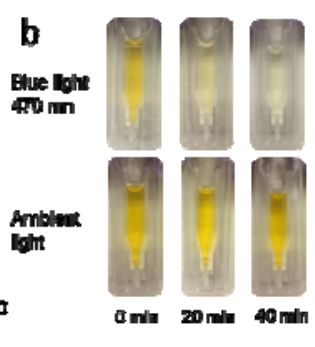

f
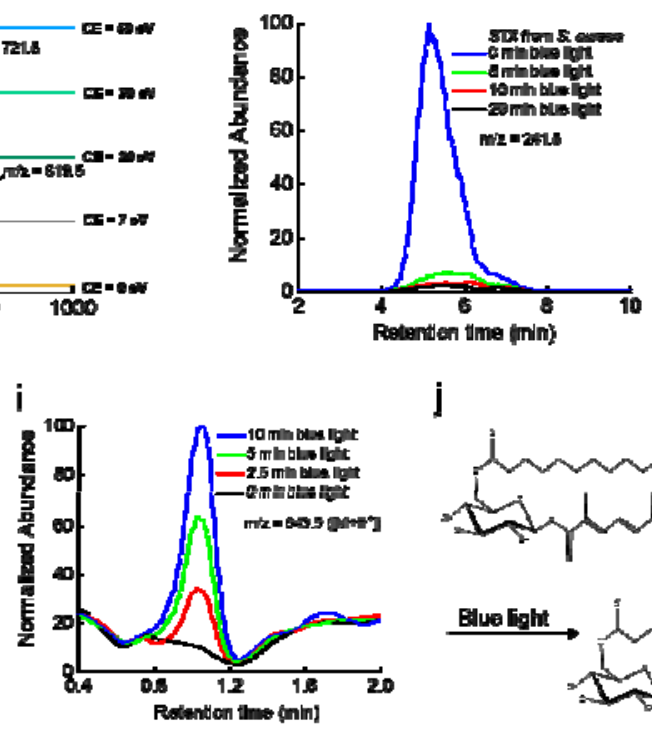

j
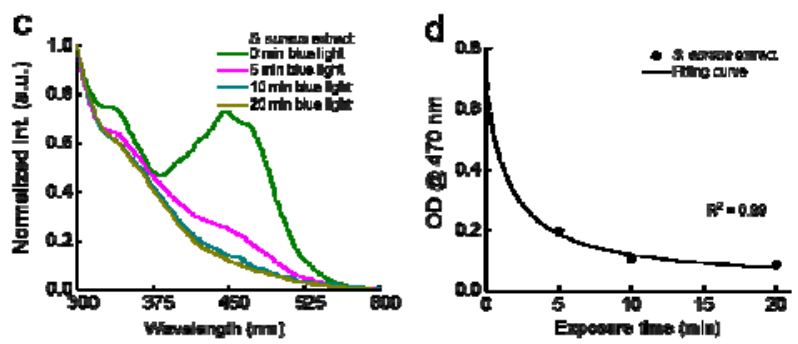

g
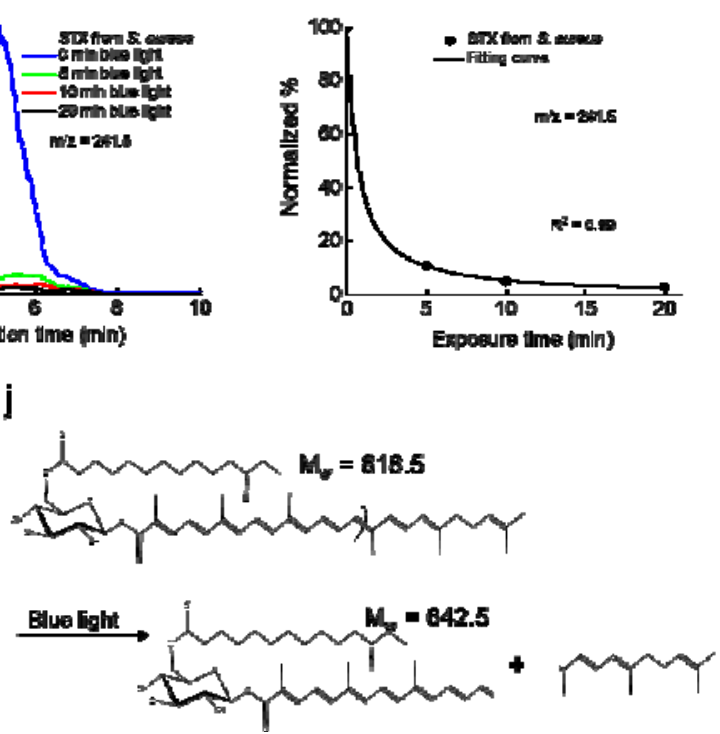

Fig. 2. Mass spectrometry unveils photochemistry of STX under blue light exposure. (a) Absorption spectra of crude STX extract (yellow) and blue light LED (black). (b) Pictures of crude STX extract exposed to blue light and ambient light with different time intervals. (c) Absorption spectra of crude STX extract over blue light exposure time. (d) OD of crude STX extract at $470 \mathrm{~nm}$ adapted from (c) over blue light exposure time. Data points are fitted by equation (1). (e) MS spectra of crude STX extract at different collision energy with peaks of $\mathrm{m} / \mathrm{z}$ $=819.5, \mathrm{~m} / \mathrm{z}=721.5$ and $\mathrm{m} / \mathrm{z}=241.5$ highlighted by black arrows. $\mathrm{CE}$, collision energy. (f) HPLC spectra of STX from untreated S. aureus over blue light exposure time. (g) The amount of STX calculated from (f) over blue light exposure time. Quantification of STX is determined from the peak area of STX in HPLC spectra. Data points are fitted by equation (1). UPLC spectra of targeted STX (h) and its corresponding product (i) over blue light exposure. (j) Representative breakdown pathway of STX by blue light indicated by (i). 

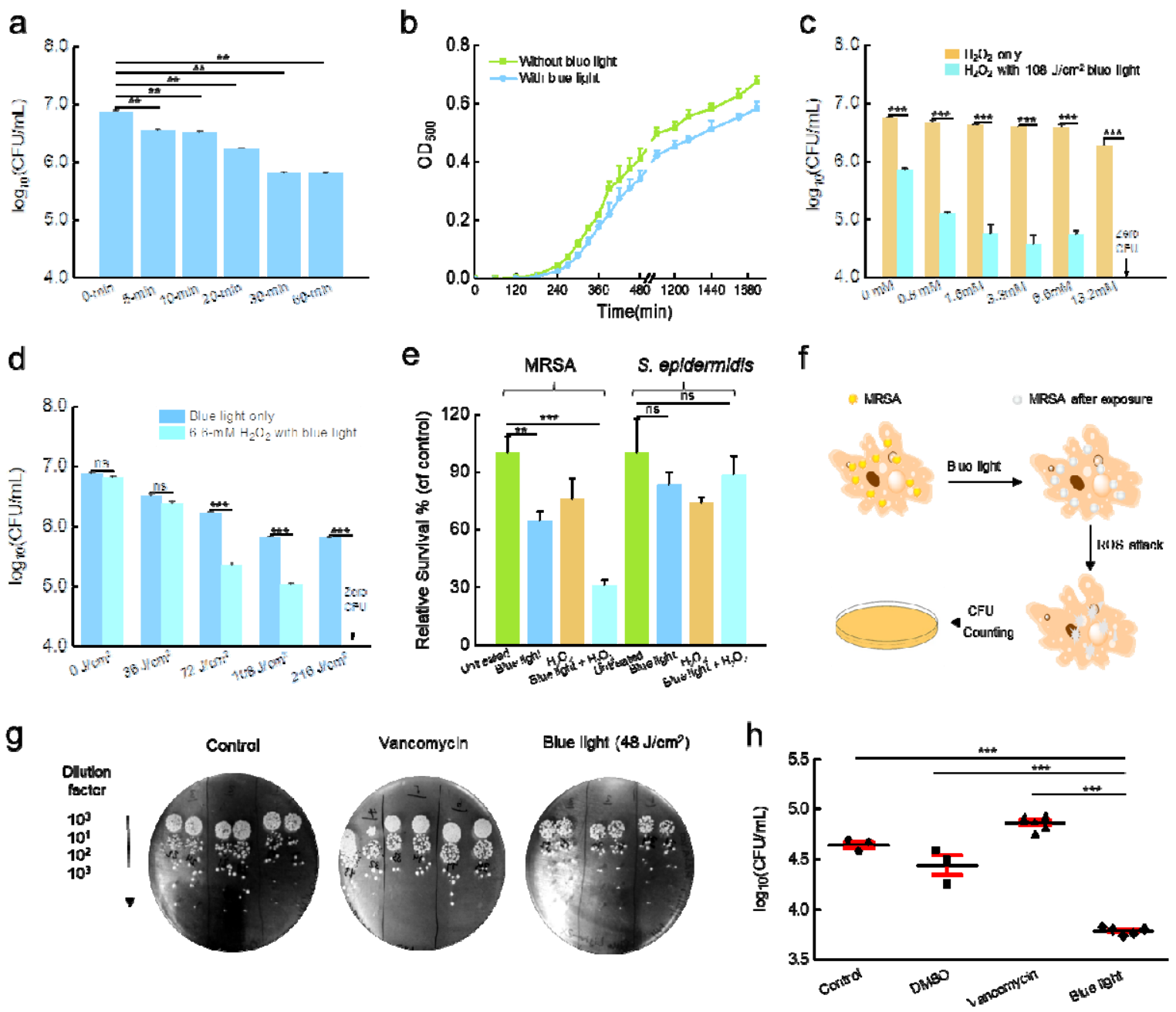

Fig. 3. Blue light and reactive oxygen species work synergistically to eliminate MRSA in culture and in macrophages. (a) MRSA CFU under blue light exposure. Blue light intensity: 60 $\mathrm{mW} / \mathrm{cm}^{2}$. (b) Growth curves of untreated and blue light-treated MRSA (culture starts at $\mathrm{t}=0 \mathrm{~min}$ immediately after the 10-min blue light exposure. Blue light dose: $120 \mathrm{~J} / \mathrm{cm}^{2}$ ). (c) MRSA CFU of $\mathrm{H}_{2} \mathrm{O}_{2}$-treated and blue light plus $\mathrm{H}_{2} \mathrm{O}_{2}$-treated groups at different $\mathrm{H}_{2} \mathrm{O}_{2}$ concentrations. (d) MRSA CFU of blue light-treated and blue light plus $\mathrm{H}_{2} \mathrm{O}_{2}$-treated groups at different blue light doses. (e) Relative survival percentage of MRSA versus S. epidermidis under different treatment schemes. Blue light dose: $60 \mathrm{~J} / \mathrm{cm}^{2}$. $\mathrm{H}_{2} \mathrm{O}_{2}: 13.2 \mathrm{mM}, 5$-min culture time. (f) Schematics illustrate the role of blue light plays in assisting macrophages to kill intracellular MRSA (not drawn to scale). (g-h) Pictures of spread plates (g) and statistical analysis (h) of CFU results of MRSAinfected macrophages from untreated, vancomycin-treated and blue light-treated groups. Error bars show standard error of the mean from at least three replicates. 
a
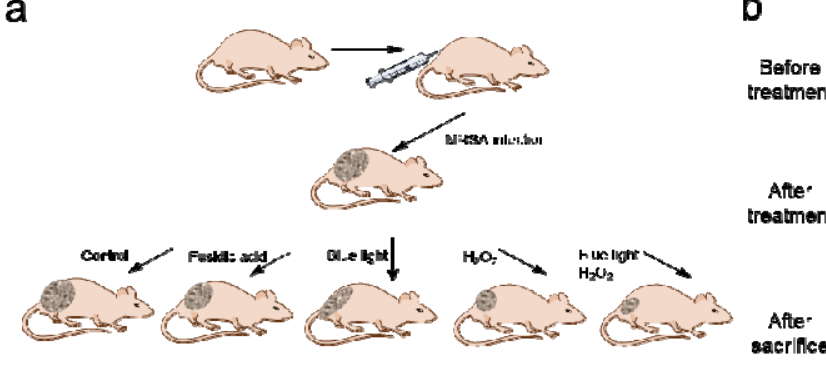

C

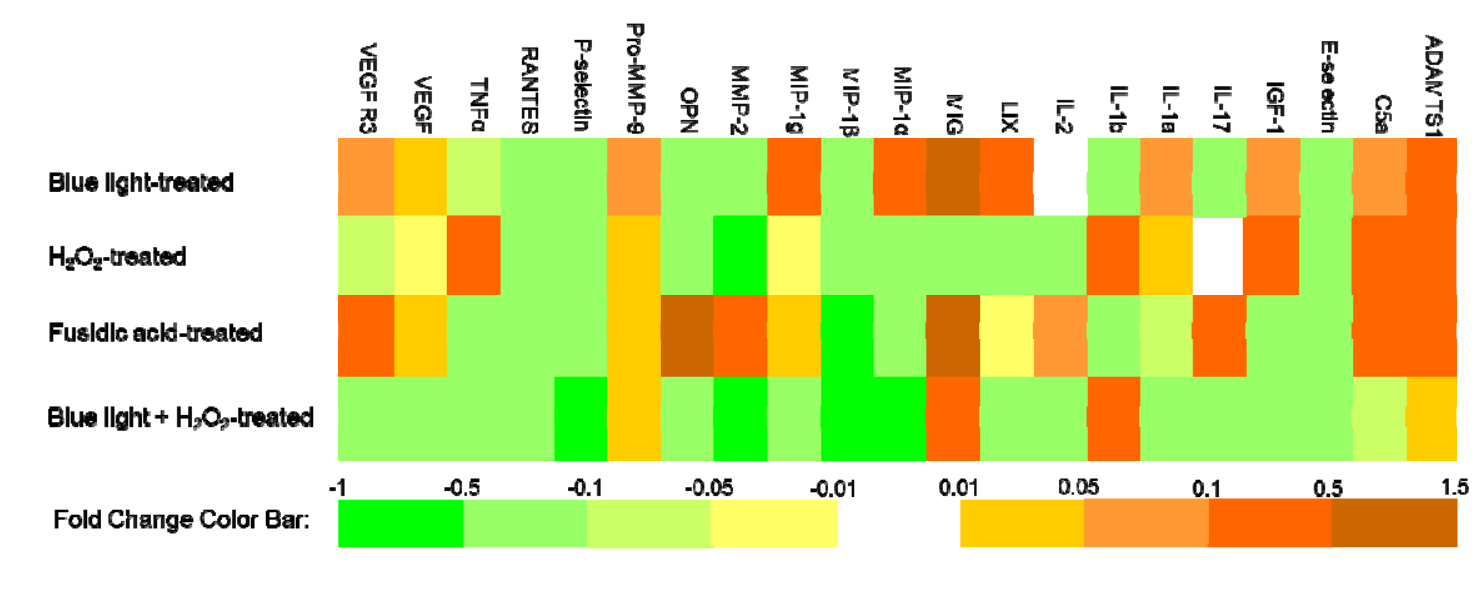

d
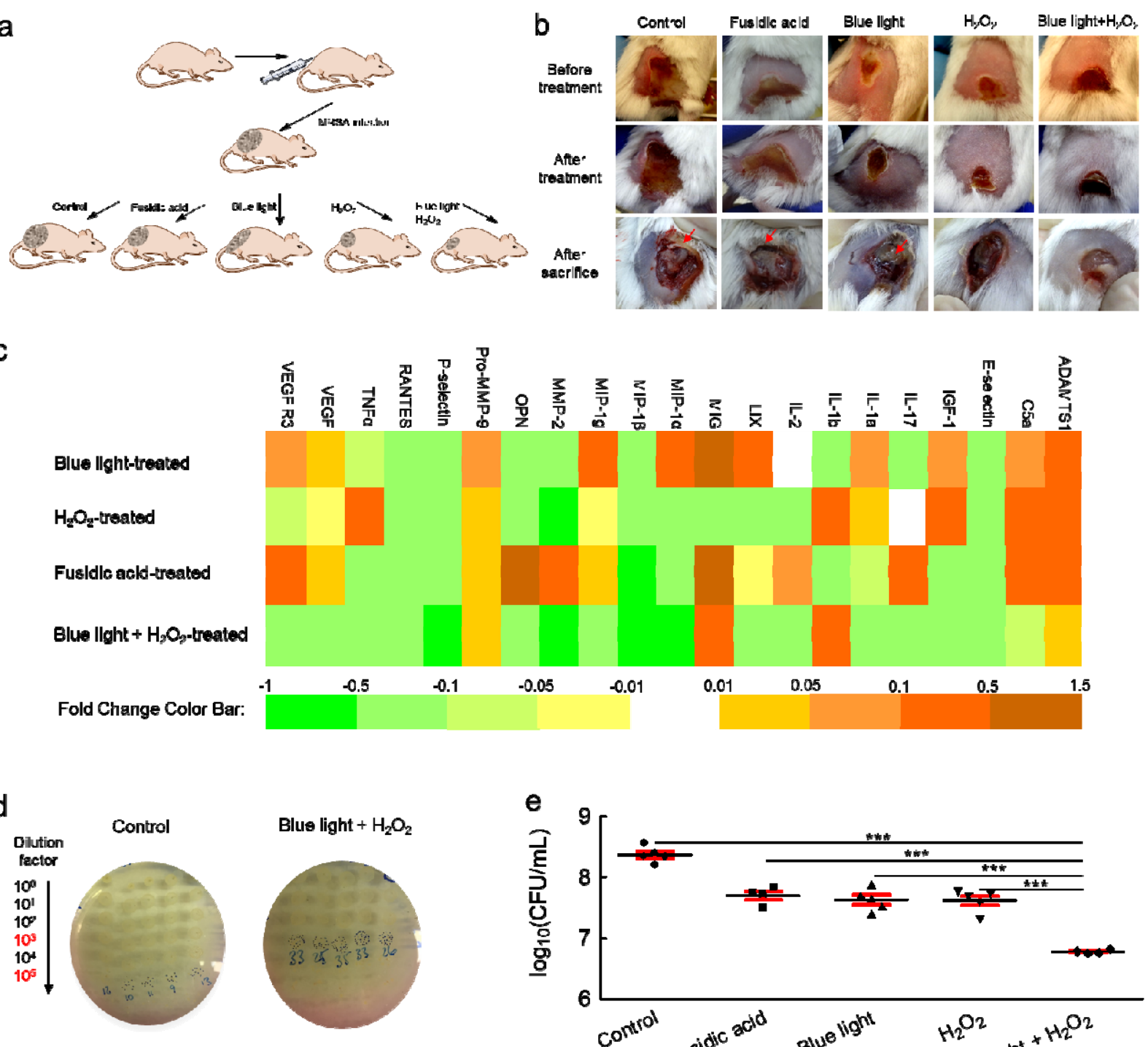

C

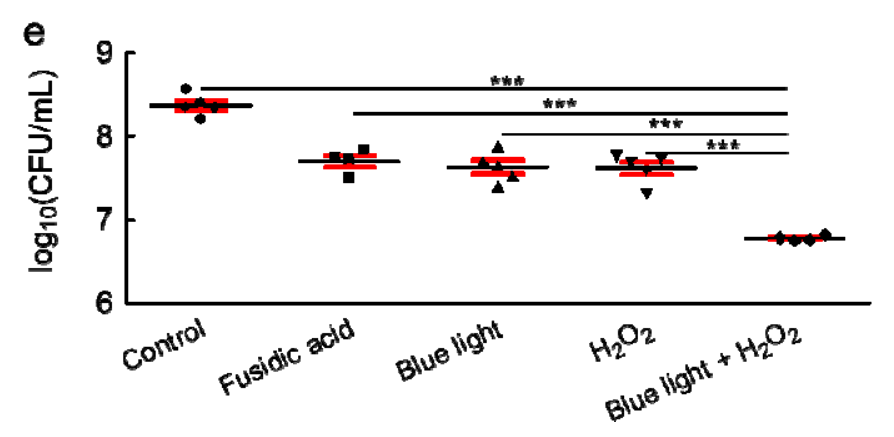

Fig. 4. Blue light plus $\mathrm{H}_{2} \mathrm{O}_{2}$ effectively reduces MRSA burden in a MRSA-infected mouse

wound. (a) Schematics of experiment design (not drawn to scale). (b) Pictures of mice wounds of five different groups taken before treatment, after treatment and after sacrifice. Red arrows indicate pus formation. (c) Heat map of key pro-inflammatory cytokines and markers expressed in tissue homogenate samples obtained from mice treated with blue light, $\mathrm{H}_{2} \mathrm{O}_{2}$, blue light plus $\mathrm{H}_{2} \mathrm{O}_{2}$, or fusidic acid. Orange box indicates upregulation; green box indicates downregulation; white indicates no significant change. (d) Pictures of spread plates from untreated and blue light plus $\mathrm{H}_{2} \mathrm{O}_{2}$-treated groups. (e) Statistical analysis of CFU results from five different groups. Error bars show the standard of the mean from $\mathrm{N}=5$ replicates. Outlier was removed through Dixon's Q test. 